

\title{
APLICAÇÃO E COMPARAÇÃO DE MÉTODOS DE APOIO À DECISÃO MULTICRITÉRIO: AHP, TODIM E PROMETHEE ॥
}

\author{
APPLICATION AND COMPARISON OF MULTI-CRITERIA DECISION SUPPORT \\ METHODS: AHP, TODIM AND PROMETHEE II
}

\begin{abstract}
iD Glauber Roger Neves ${ }^{1}$
iD Antonio Cesar Galhardi²

Wagner Cezar Lucato ${ }^{3}$
\end{abstract}

1 Mestre em Gestão e Tecnologia em Sistemas Produtivos

Centro Estadual de Educação Tecnológica Paula Souza - CEETEPS.

São Paulo, SP - Brasil.

7glaub@gmail.com

${ }^{2}$ Doutor em Engenharia Mecânica

Centro Estadual de Educação Tecnológica Paula Souza - CEETEPS.

São Paulo, SP - Brasil.

galhardi.antoniocesar@gmail.com

${ }^{3}$ Doutor em Engenharia de Produção Universidade Nove de Julho - UNINOVE.

São Paulo, SP - Brasil.

wlucato@uni9.pro.br

Recebido em: 25 jun. 2020

Aprovado em: 29 ago. 2020
Resumo: Uma das críticas a respeito dos métodos multicritérios é que diferentes técnicas podem produzir resultados discordantes quando aplicadas ao mesmo problema. Neste contexto, o objetivo desta pesquisa é comparar os métodos de apoio à tomada de decisão multicritério AHP, TODIM e PROMETHEE II. Para alcançar este propósito, os métodos foram aplicados a um processo de seleção de software mediante sete fornecedores qualificados. Além de verificar e comparar a consistência dos resultados, utilizando-se de grupos contendo 3, 7 e 11 critérios, este estudo também apresenta uma percepção da aplicabilidade, complexidade, e laborosidade requerida do usuário quanto à utilização dos métodos. Embora os três métodos se baseiem em diferentes paradigmas, a metodologia de aplicação utilizada possibilitou o apontamento de características individuais e de pontos positivos e negativos de um método sobre o outro. Com respeito à consistência dos resultados, constatou-se a ocorrência de algumas inversões na ordem de classificação dos fornecedores.

Palavras-chave: Métodos multicritérios. AHP. TODIM. PROMETHEE II.

Abstract: One of the criticisms about multi-criteria methods is that different techniques could produce discordant results when applied to the same problem. In this context, the aim of this research is to compare the methods of multiple-criteria decision making AHP, TODIM and PROMETHEE II. To achieve this goal, the methods were applied to a software selection process through seven qualified suppliers. In addition to verifying and comparing the consistency of the results, using groups with 3, 7 and 11 criteria, this study also presents a perception of the applicability, complexity, and laborability required of the user regarding to the execution of the methods. Although the three methods are based on different paradigms, the application methodology allowed the identification of individual characteristics and positive and negative points of one method over the other. With respect to the consistency of the results, it was verified the occurrence of some inversions in the order of classification of the suppliers.

Keywords: Multi-criteria methods. AHP. TODIM. PROMETHEE II. 


\section{Introdução}

Nas últimas décadas, o crescente número de informações e a necessidade de utilizá-las em um processo decisório complexo contribuiu para o surgimento de diversos métodos de apoio à tomada de decisão multicritério (MCDM). Estes métodos são eficazes para a resolução de problemas em que ocorram critérios conflitantes (Brans \& Mareschal, 2005).

De modo geral, os métodos MCDM se diferem por suas limitações, adequação ao contexto de uso e a forma de abordagem das peculiaridades do problema (Ho, Xu \& Dey, 2010). Todos os métodos têm deficiências, suposições, níveis de sofisticação e outras características distintas (Ishizaka \& Nemery, 2013). A pesquisa realizada por Qaradagui (2016) sustenta que provavelmente não exista uma maneira de se verificar a exatidão dos métodos MCDM, e nem de constatar um modelo soberano. As duas únicas formas disponíveis para avaliar o desempenho dos métodos MCDM são os estudos empíricos e por meio de comparação. Como é mais desafiador a realização de estudos empíricos, além de seus fatores subjetivos e de erros técnicos, a comparação dos métodos pode ser considerada mais razoável (Selmi, Kormi \& Ali, 2013).

Em decorrência das oportunidades expostas, o problema da elaboração deste trabalho envolve o seguinte questionamento: Quais seriam os resultados da utilização de três métodos multicritérios diferentes em relação à percepção dos parâmetros de: aplicabilidade; consistência; complexidade; e laborosidade, quando se considera um mesmo problema de decisão? A partir do problema abordado, o objetivo desta pesquisa é comparar os métodos de apoio à tomada de decisão multicritério AHP, TODIM e PROMETHEE II. Para alcançar esse objetivo, os métodos foram aplicados a um problema de seleção de um software, mediante fornecedores qualificados.

O procedimento técnico aplicado nesta pesquisa, utilizando-se especificamente de três métodos MCDM, surge como uma alternativa na construção de uma estrutura analítica para a sistematização do processo decisório de seleção, levando em consideração diversos critérios de preferência.

\section{Referencial teórico}

As abordagens multicritérios são formas de modelar os processos de escolha que envolve: uma decisão a ser tomada; as ocorrências desconhecidas que podem interferir nos resultados; as possíveis trajetórias de ação; e os respectivos resultados. Estes modelos demonstram, de maneira aceitável, o juízo de valores dos envolvidos. Assim, os métodos multicritérios agem como uma base para argumentos, principalmente nos eventos nos quais existem conflitos entre os tomadores de decisão e também quando a percepção do problema pelos vários interessados ainda não está totalmente estabelecida (Noronha, 1998). Com a aplicação dos métodos multicritérios, o tomador de decisão pode 
ser capaz de presumir as possíveis implicações de cada curso de ação, de modo a obter uma melhor compreensão das vinculações entre suas ações e seus objetivos (Flament, 1999).

Duas escolas se destacam com relação aos métodos MCDM, estas se tornaram referências e pilares para o desenvolvimento de qualquer novo método: a escola americana e a escola francesa. Os principais métodos da escola americana são o AHP (Analytic Hierarchy Process) e o MAUT (Multiattribute Utility Theory). Já na escola francesa se destacam as famílias ELECTRE (Elimination Et Choix Traduisant la Réalité) e o PROMETHEE (Preference Ranking Method for Enrichment Evaluation) (Bhushan \& Rai, 2004; Gomes, 2007). De acordo com Gomes e Rangel (2009), o método TODIM (Tomada de Decisão Interativa Multicritério) é considerado um modelo híbrido que combina aspectos da abordagem americana e francesa.

\subsection{AHP}

O método AHP foi elaborado por Saaty na década de 70. O método consiste em uma metodologia de suporte à decisão multicritério que objetiva a seleção ou ordenação de alternativas, em um processo que considera diferentes critérios de avaliação.

Muitos autores têm desenvolvido suas pesquisas utilizando o método AHP na resolução de problemas de tomada de decisão nas mais diversas áreas. Muralidharan, Anantharaman, e Deshmukh (2002) propuseram um modelo de cinco etapas do AHP para auxiliar os tomadores de decisão na classificação e seleção dos fornecedores com relação a nove critérios de avaliação. Padovani, Carvalho e Muscat (2010) aplicaram o método na escolha de alternativas para integrar o portfólio de projetos de uma empresa química. Trendowicz e Kopczynska (2014) analisaram 18 aplicações de métodos MCDM em problemas de avaliação da qualidade de software e constataram que uma ampla diversidade de métodos vem sendo utilizada, porém segundo os autores, o método AHP é o mais utilizado no apoio a este tipo de problema.

\subsection{TODIM}

Com relação ao método TODIM, Gomes (2007) expressou que este foi concebido em sua forma atual no início dos anos 90 e é provavelmente o único método multicritério fundamentado na Teoria dos Prospectos. A Teoria dos Prospectos foi desenvolvida a partir das pesquisas de Kahneman e Tversky (1979), que avaliaram o comportamento humano durante a tomada de decisões em situações de risco. Para expor a aversão e a propensão ao risco.

A pesquisa de Longaray, Popiolek Jr., Munhoz, Geri e Castelli (2015) não encontrou um número relevante de estudos envolvendo a produção científica aplicada ao método TODIM, todavia os autores que abordaram o tema desenvolveram suas pesquisas nas mais diversas áreas. Sen, Datta, e Mahapatra 
(2016) aplicaram o método TODIM para seleção de um modelo de robô industrial. Zindani, Maity, Bhowmik e Chakraborty (2017) utilizaram o TODIM para selecionar os materiais mais adequados para a fabricação de duas peças automobilísticas. Dehghani, Siami e Haghi (2017) aplicaram o modelo para definir o melhor método de mineração em uma mina de ferro no Irã. Rangel e Gomes (2007) apresentaram um estudo visando auxiliar na definição do valor de referência de aluguel de imóveis residenciais. Ao empregarem o método TODIM para realizar a ordenação dos imóveis com diferentes características, chegou-se às diversas faixas de valores de aluguel dos imóveis em estudo.

\subsection{PROMETHEE}

Em meados dos anos 80, Brans, Vincke e Mareschal apresentaram o método PROMETHEE como uma nova classe de método de análise multicritério. O método PROMETHEE, tem aplicação em diversas áreas de conhecimento e pertence à família de métodos de sobreclassificação (outranking). É baseado em uma relação binária entre as ações ou alternativas e as principais características deste método são a simplicidade, clareza, estabilidade e flexibilidade (Brans \& Mareschal, 2005).

O método PROMETHEE tem sido aplicado em diversas áreas. Doukas, Patlitzianas, e Psarras (2006) aplicaram o PROMETHEE II para avaliar as tecnologias sustentáveis para geração de eletricidade de acordo com fatores ambientais, sociais e econômicos. Dulmin e Mininno (2003) utilizaram o método para escolher fornecedores relevantes para uma empresa italiana de médio porte que opera no campo de transporte rodoviário e ferroviário. Lim, Ayoko, Morawska, Ristovski e Jayaratne (2007) utilizaram o PROMETHEE II para classificar fatores de emissões de veículos com relação à tipos de combustíveis e parâmetros de operações dos motores.

\subsection{Aplicação conjunta de métodos MCDM}

Com o objetivo de aumentar o nível de confiabilidade quanto ao processo de tomada de decisão, alguns estudos aplicaram simultaneamente múltiplos métodos MCDM para o mesmo problema de decisão. Existe pouca ocorrência de estudos comparativos entre os métodos multicritérios, devido às diversas possibilidades de comparação mediante a grande pluralidade de métodos existentes (Lima Junior, 2013). Desta maneira, na literatura pode ser encontrado alguns estudos que comparam ou mesclam os métodos MCDM considerando o contexto de problemas específicos.

Efe (2016), Gurbuz, Alptekin e Alptekin (2012), e Kilic, Zaim e Delen (2015) aplicaram variados métodos MCDM com o intuito de priorizar softwares. Outros trabalhos como o de Anojkumar, Ilangkumaran e Sasirekha (2014), Hodgett (2016), Onut, Kara e Isik (2009), Passos e Gomes (2005) e Ribeiro, Passos e Teixeira (2012) tiveram o propósito de selecionar fornecedor, materiais ou equipamentos por meio da utilização de múltiplos métodos MCDM. Em alguns casos, quando os 
métodos foram comparados, algumas pesquisas produziram resultados semelhantes, enquanto outras obtiveram variações entre os resultados. Todos os estudos foram conduzidos a partir da realização de uma pesquisa bibliográfica seguida pelo desenvolvimento de modelos de decisão e por aplicação dos métodos. Além dos procedimentos de pesquisa utilizados, outra característica comum a esses estudos é a adoção de parâmetros de comparação bem definidos. Exemplos desses parâmetros incluem a complexidade dos métodos, procedimentos para a avaliação de alternativas e consistência dos resultados fornecidos.

\section{Método}

O objetivo deste trabalho é comparar três distintos métodos de apoio à tomada de decisão. De acordo com Ishizaka e Labib (2009), três é o número mínimo exigido de resultados de ranking de conjuntos que podem ser comparados uns com os outros. Segundo os mesmos autores, com um único método é impossível fornecer uma análise efetiva e realista devido às suas limitações e suposições inerentes.

Para a aplicação dos métodos MCDM, é fundamental a identificação dos critérios e alternativas a serem estudados. Com relação às alternativas, ou seja, os fornecedores a serem classificados por meio da aplicação dos métodos MCDM, definiu-se pela utilização de sete fornecedores de um software de simulação específica, denominados por S1, S2, S3, S4, S5, S6 e S7. Quanto aos critérios, estes podem ser definidos como os padrões que precisam ser levados em consideração para uma avaliação dos fornecedores. A lista de critérios depende das características contextuais do problema e, portanto, pode variar de um estudo para outro. Neste trabalho, os critérios foram definidos com base em uma revisão sistemática da literatura, verificando-se pesquisas que utilizaram critérios comuns para a seleção de um software. Com isto, foram identificados 11 critérios comuns, e estes foram alocados em três grupos: o Grupo K com os três primeiros critérios; o Grupo W com os sete primeiros critérios; e o Grupo Y com 11 critérios, de acordo com o mostrado no Quadro 1. Cada grupo de critérios, K, W e Y, foi utilizado na aplicação dos métodos MCDM.

\section{Quadro 1}

Grupos de critérios

\begin{tabular}{|c|c|c|c|c|c|}
\hline Grupo K & \multicolumn{2}{|c|}{ Grupo W } & \multicolumn{3}{|c|}{ Grupo Y } \\
\hline - Critério 1 & - Critério 1 & - Critério 4 & - Critério 1 & - Critério 4 & - Critério 8 \\
\hline - Critério 2 & - Critério 2 & - Critério 5 & - Critério 2 & - Critério 5 & - Critério 9 \\
\hline - Critério 3 & - Critério 3 & $\begin{array}{l}\text { - Critério } 6 \\
\text { - Critério } 7\end{array}$ & - Critério 3 & $\begin{array}{l}\text { - Critério } 6 \\
\text { - Critério } 7\end{array}$ & $\begin{array}{l}\text { - Critério } 10 \\
\text { - Critério } 11\end{array}$ \\
\hline
\end{tabular}

Fonte: Os Autores. 
Após a definição dos critérios e alternativas, O AHP, o TODIM e o PROMETHEE ॥ foram computados por meio do editor de planilhas Microsoft Excel. A execução dos cálculos seguiu as etapas estabelecidas nos procedimentos de cálculo dos métodos. Foram determinadas três rodadas de cálculos para cada método MCDM, utilizando os grupos de critérios K, W, e Y. Nesta etapa também se verificou a consistência dos resultados obtidos pelos três métodos.

Além disto, os três métodos MCDM foram comparados e discutidos quanto à percepção de:

- Aplicabilidade, ou seja, os três métodos são realmente aptos para a resolução de problema de classificação de fornecedores?

- Consistência das classificações de ordenações, ou seja, os três métodos apresentam os mesmos resultados na ordem das classificações?

- Complexidade, ou seja, quão complexo é aplicar cada método?

- Laboriosidade, ou seja, quão numerosos são os cálculos para obtenção dos resultados? Qual método demanda maior tempo e empenho para a obtenção dos resultados?

Entre outros fatores, que permitiram avaliar e apontar aspectos específicos de cada um dos três métodos MCDM.

\section{Resultados e discussão}

Neste trabalho, foram simuladas três rodadas de cálculos para cada método MCDM, utilizando os grupos de critérios K (3 critérios), W (7 critérios) e Y (11 critérios) e também os fornecedores S1, S2, S3, S4, S5, S6 e S7. As classificações obtidas com as aplicações dos métodos multicritérios AHP, TODIM e PROMETHEE foram verificadas e comparadas entre si. A Tabela 1 demonstra a classificação obtida na execução de cada um dos métodos para o Grupo K, com três critérios.

\section{Tabela 1}

Resultados da aplicação dos métodos MCDM (Grupo K)

\begin{tabular}{|c|c|c|c|c|c|c|}
\hline Fornecedor & \multicolumn{2}{|c|}{ AHP } & \multicolumn{2}{|c|}{ TODIM } & \multicolumn{2}{|c|}{ PROMETHEE II } \\
\hline S1 & 0,192 & 20 & 0,793 & 20 & 0,311 & $2 \circ$ \\
\hline S2 & 0,112 & 5응 & 0,468 & 50 & $-0,526$ & 60 \\
\hline S3 & 0,137 & 3으 & 0,633 & $3 / 4 ㅇ$ & 0,211 & 3으 \\
\hline S4 & 0,109 & 6o & 0,325 & 6o & $-0,063$ & 5 \\
\hline S5 & 0,056 & 70 & 0,000 & 70 & $-0,621$ & 70 \\
\hline S6 & 0,263 & 10 & 1,000 & 10 & 0,531 & 10 \\
\hline S7 & 0,131 & 40 & 0,633 & $3 / 4 ㅇ$ & 0,157 & 40 \\
\hline
\end{tabular}

Fonte: Os Autores. 
Observando-se a Tabela 1, percebe-se que as análises dos fornecedores efetuadas por cada um dos três métodos MCDM conduziram essencialmente à mesma classificação para as quatro melhores posições. Dessa forma, os fornecedores S6, S1, S3 e S7, apresentam posições coincidentes, sendo que o método TODIM calculou o mesmo valor de utilidade global para os fornecedores S3 e S7. Na quinta e sexta posições, a classificação se manteve para os métodos AHP e TODIM, com o fornecedor S2 na quinta posição e o fornecedor S4 na sexta posição. Porém, no método PROMETHEE II, essas posições se inverteram. O fornecedor S5 foi classificado na última posição pelos três métodos MCDM. Portanto, em uma decisão racional, quando considerados os critérios do Grupo K e apoiando-se na tomada de decisão multicritério, a melhor alternativa seria a escolha do fornecedor $\mathrm{S6}$.

A Tabela 2 demonstra a classificação obtida na execução de cada um dos métodos para o Grupo W, com sete critérios.

Tabela 2

Resultados da aplicação dos métodos MCDM (Grupo W)

\begin{tabular}{|c|c|c|c|c|c|c|}
\hline Fornecedor & \multicolumn{2}{|c|}{ AHP } & \multicolumn{2}{|c|}{ TODIM } & \multicolumn{2}{|c|}{ PROMETHEE II } \\
\hline S1 & 0,239 & 10 & 1,000 & 10 & 0,590 & 10 \\
\hline s2 & 0,136 & 40 & 0,451 & $3 ㅇ$ & $-0,129$ & 40 \\
\hline S3 & 0,199 & 20 & 0,725 & 20 & 0,302 & 20 \\
\hline S4 & 0,087 & 60 & 0,101 & 60 & $-0,237$ & 60 \\
\hline S5 & 0,080 & 7음 & 0,000 & 7음 & $-0,549$ & 70 \\
\hline S6 & 0,165 & 30 & 0,229 & 40 & 0,211 & 30 \\
\hline S7 & 0,093 & 50 & 0,112 & 50 & $-0,189$ & 50 \\
\hline
\end{tabular}

Fonte: Os Autores.

De acordo com a Tabela 2, constata-se que as análises das alternativas efetuadas por cada um dos três métodos MCDM conduziram essencialmente à mesma classificação para as duas melhores posições. Dessa forma, os fornecedores S1 e S3, apresentam posições coincidentes. Na terceira e quarta posições, a classificação se manteve para os métodos AHP e PROMETHEE II, com o fornecedor S6 na terceira posição e o fornecedor S2 na quarta posição. Todavia, no método TODIM essas posições se inverteram. Para as três últimas colocações, os métodos tiveram comportamento iguais. O fornecedor S7 foi classificado na quinta posição, S4 na sexta posição e S5 na última colocação. Portanto, em uma decisão racional, quando considerados os critérios do Grupo W e apoiando-se na tomada de decisão multicritério, a melhor alternativa seria a escolha do fornecedor S1.

A Tabela 3 demonstra a classificação obtida na execução de cada um dos métodos para o Grupo $\mathrm{Y}$, com onze critérios. 


\section{Tabela 3}

Resultados da aplicação dos métodos MCDM (Grupo Y)

\begin{tabular}{|c|c|c|c|c|c|c|}
\hline Fornecedor & \multicolumn{2}{|c|}{ AHP } & \multicolumn{2}{|c|}{ TODIM } & \multicolumn{2}{|c|}{ PROMETHEE II } \\
\hline S1 & 0,215 & 20 & 1,000 & 10 & 0,427 & 10 \\
\hline s2 & 0,144 & 30 & 0,453 & 3응 & 0,035 & 40 \\
\hline S3 & 0,223 & 10 & 0,741 & 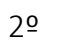 & 0,409 & $2 \circ$ \\
\hline S4 & 0,115 & 50 & 0,070 & 50 & $-0,084$ & 50 \\
\hline S5 & 0,074 & 70 & 0,000 & $7 \underline{0}$ & $-0,580$ & 70 \\
\hline 56 & 0,143 & 40 & 0,372 & $4 \stackrel{0}{2}$ & 0,057 & 3은 \\
\hline S7 & 0,086 & 60 & 0,064 & 6음 & $-0,264$ & 60 \\
\hline
\end{tabular}

Fonte: Os Autores.

Segundo mostra a Tabela 3, verifica-se que pela primeira vez os fornecedores melhores classificados não foram coincidentes para os três métodos MCDM. Na primeira e segunda posições, a classificação se manteve para os métodos TODIM e PROMETHEE II, com o fornecedor S1 na primeira posição e o fornecedor S3 na segunda posição. Porém, no método AHP essas posições se inverteram. Com relação aos fornecedores S2 e S6, estes, de acordo com os cálculos do AHP e do TODIM, se classificaram na terceira e quarta posição. Porém, no método PROMETHEE II essas posições se inverteram. Para as três últimas colocações, os métodos MCDM tiveram comportamento iguais. O fornecedor S4 foi classificado na quinta posição, S7 na sexta posição e S5 ficou na última colocação. Desta vez, devido à inversão dos resultados obtidos pelo método AHP para as duas melhores classificações, quando comparados aos métodos TODIM e PROMETHEE, não é possível afirmar com exatidão se é o fornecedor S1 ou o fornecedor S3 a melhor alternativa para escolha.

Com a aplicação e estudo dos três métodos MCDM, tornou-se possível a análise dos pontos positivos e pontos negativos de cada ferramenta. O Quadro 2 resume alguns parâmetros de comparação como aplicabilidade do método, ordenação das classificações dos fornecedores, complexidade do método, laboriosidade para a obtenção dos resultados, entre outros, que permitiram avaliar e apontar aspectos percebidos durante a elaboração deste estudo. 
Quadro 2

Parâmetros de comparação dos métodos MCDM

\begin{tabular}{|c|c|c|c|c|}
\hline \multirow[b]{2}{*}{ Aplicabilidade } & \multirow{5}{*}{$\begin{array}{l}\text { Grupo K } \\
\text { (3 critérios) } \\
\text { Grupo W } \\
\text { (7 critérios) } \\
\text { Grupo Y } \\
\text { (11 critérios) }\end{array}$} & AHP & TODIM & PROMETHEE II \\
\hline & & apto & apto & apto \\
\hline \multirow{3}{*}{$\begin{array}{l}\text { Ordem de } \\
\text { classificação } \\
\text { dos } \\
\text { fornecedores }\end{array}$} & & $\begin{array}{c}\mathrm{S} 6>\mathrm{S} 1>\mathrm{S} 3 \text { e } \mathrm{S} 7>\mathrm{S} 2> \\
\mathrm{S} 4>\mathrm{S} 5\end{array}$ & $\begin{array}{c}\mathrm{S} 6>\mathrm{S} 1>\mathrm{S} 3 \text { e } \mathrm{S} 7>\mathrm{S} 2> \\
\mathrm{S} 4>\mathrm{S} 5\end{array}$ & $\begin{array}{c}\mathrm{S} 6>\mathrm{S} 1>\mathrm{S} 3>\mathrm{S} 7>\mathrm{S} 4> \\
\mathrm{S} 2>\mathrm{S} 5\end{array}$ \\
\hline & & $\begin{array}{c}\mathrm{S} 1>\mathrm{S} 3>\mathrm{S} 6 \text { e } \mathrm{S} 2>\mathrm{S} 7> \\
\mathrm{S} 4>\mathrm{S} 5\end{array}$ & $\begin{array}{c}\mathrm{S} 1>\mathrm{S} 3>\mathrm{S} 2>\mathrm{S} 6>\mathrm{S} 7> \\
\mathrm{S} 4>\mathrm{S} 5\end{array}$ & $\begin{array}{c}\mathrm{S} 1>\mathrm{S} 3>\mathrm{S} 6>\mathrm{S} 2>\mathrm{S} 7> \\
\mathrm{S} 4>\mathrm{S} 5\end{array}$ \\
\hline & & $\begin{array}{c}\mathrm{S} 3>\mathrm{S} 1>\mathrm{S} 2 \text { e } \mathrm{S} 6>\mathrm{S} 4> \\
\mathrm{S} 7>\mathrm{S} 5\end{array}$ & $\begin{array}{c}\mathrm{S} 1>\mathrm{S} 3>\mathrm{S} 2>\mathrm{S} 6>\mathrm{S} 4> \\
\mathrm{S} 7>\mathrm{S} 5\end{array}$ & $\begin{array}{c}\mathrm{S} 1>\mathrm{S} 3>\mathrm{S} 6>\mathrm{S} 2>\mathrm{S} 4> \\
\mathrm{S} 7>\mathrm{S} 5\end{array}$ \\
\hline \multicolumn{2}{|l|}{ Complexidade } & baixa & alta & média \\
\hline \multicolumn{2}{|l|}{ Laboriosidade } & alta & média & média \\
\hline \multicolumn{2}{|c|}{ Qtde. de critérios } & $\begin{array}{l}\text { recomenda-se um } \\
\text { limite de nove (9) }\end{array}$ & sem limite & sem limite \\
\hline \multirow{2}{*}{\multicolumn{2}{|c|}{$\begin{array}{l}\text { Aplicação na literatura } \\
\text { Software de suporte }\end{array}$}} & alta frequência & baixa frequência & baixa frequência \\
\hline & & alta disponibilidade & indisponível & média disponibilidade \\
\hline
\end{tabular}

Quanto à aplicabilidade dos métodos MCDM, todos os três métodos se revelaram ferramentas de apoio aptas para a elaboração de uma classificação ordenada de fornecedores, considerando-se os critérios e alternativas identificados neste estudo. No método AHP, a classificação das alternativas é determinada pelos resultados das prioridades compostas. No método TODIM, a classificação é obtida pelos valores de utilidade global das alternativas, que medem seus valores de ganhos e perdas. Já no método PROMETHEE II, a colocação das alternativas é originada conforme os resultados dos valores dos fluxos líquidos.

De modo geral, a ordem de classificação dos fornecedores estabelecidos pelos três métodos multicritérios se mostraram consistentes quando comparados um a outro, uma vez que, analisando sete possibilidades de fornecedores, ocorreram poucas inversões de colocações. É válido observar que quando se ocorreu inversões, as alternativas invertidas se localizavam próximas entre si, a apenas uma colocação acima ou a uma colocação abaixo. Para o grupo com três critérios (Grupo K), os resultados do método AHP e do método TODIM foram integralmente concordantes. O método PROMETHEE II teve apenas uma inversão entre as posições. Em outra avaliação, com referência ao grupo com sete critérios (Grupo W), as classificações resultantes do método AHP e do método PROMETHEE II revelaram-se integralmente concordantes. O método TODIM teve apenas uma inversão entre as posições. No entanto, quando considerado o grupo com onze critérios (Grupo Y), o método AHP apresentou resultados discordantes importantes, como a classificação invertida para o primeiro e segundo colocados em comparação aos métodos TODIM e PROMETHEE. Esse resultado desigual obtido pelo método AHP, levanta dúvidas sobre a utilização do método AHP quando se têm mais de nove critérios. As classificações resultantes do método TODIM revelaram-se as mais concordantes em comparação aos outros métodos. Os métodos MCDM desempenham uma função de apoio à tomada de decisão, e, 
considerando os resultados obtidos para os três grupos de critérios, a escolha de um fornecedor deveria se restringir entre o primeiro e segundo melhores classificados.

Na ocasião em que os métodos AHP, TODIM e PROMETHEE II são comparados em relação à complexidade dos cálculos, o TODIM se qualificou como o mais complicado devido à aplicação de suas equações. O método AHP tem uma metodologia menos complexa em relação ao TODIM, porém requer o entendimento de alguns conceitos matemáticos, como autovalor e autovetor. O procedimento matemático utilizado pelo PROMETHEE II é relativamente fácil de se compreender, pois a elaboração da classificação das alternativas é baseada em uma simples teoria de concordância e discordância; todavia, o grau de complexidade dos cálculos do PROMETHEE II é vinculado a como o tomador de decisão define a função de preferência de cada critério.

Quando os métodos AHP, TODIM e PROMETHEE II são comparados em relação à laborosidade, observou-se que o AHP requer um maior número de cálculos devido às múltiplas verificações paritárias necessárias para as matrizes de comparação. Com a utilização de uma planilha Excel, verificou-se também que a quantidade de cálculos necessários para a aplicação do método AHP é proporcional à quantidade de critérios e alternativas envolvidos. Com referência aos métodos TODIM e PROMETHEE II, na percepção deste estudo, a quantidade de cálculos se equivalem, contudo, não podem ser considerados métodos com baixa quantidade de cálculos, devido à utilização de diversas equações em seus procedimentos. A demanda de tempo necessários para a aplicação dos métodos MCDM são diretamente relacionados aos procedimentos e às quantidades de cálculos exigidos pelos métodos. Portanto, neste parâmetro de comparação, o método AHP requer um maior esforço e gasto de tempo do usuário para se chegar a um resultado quando confrontado com os outros dois métodos. Esta análise foi baseada na aplicação dos métodos utilizando-se do Microsoft Excel e, possivelmente, com a utilização de ferramentas de software, a percepção destes parâmetros poderá ser discordante.

Quando os métodos AHP, TODIM e PROMETHEE II são comparados em relação ao efeito da quantidade de critérios na variação das classificações, verificou-se que quando é considerado o grupo com três critérios, apenas o método PROMETHEE II apresentou uma inversão dos fornecedores. Caso considerado o grupo com sete critérios, apenas o método TODIM apresentou uma alteração na ordem de classificação. As inversões ocorridas nos grupos com três ou sete critérios não comprometeram os resultados finais, pois para as alternativas melhores colocadas, não ocorreram variações nas classificações. Entretanto, caso seja considerado o grupo com 11 critérios, as inversões mais importantes ocorreram com o AHP, que divergiu as classificações dos primeiros colocados. Neste contexto, é fundamental mencionar que o método AHP requer alguma limitação no número de critérios e alternativas. Saaty (1980), indicou que o número de alternativas ou critérios de decisão para ser comparado usando o AHP deve ser limitado a nove, de modo a não comprometer os julgamentos e sua 
consistência. Os métodos TODIM e PROMETHEE II não requerem nenhum limite no número de critérios ou alternativas no processo de tomada de decisão.

Quanto à definição dos pesos, os métodos AHP, TODIM e PROMETHEE II também são distintos. No AHP, para a determinação dos pesos, durante os julgamentos, o seguinte questionamento precisa ser respondido: "Quão importante é a opção A em relação à opção B na contribuição para um objetivo?". Com base em uma sequência de comparações paritárias, as prioridades relativas (pesos) são determinadas usando o parâmetro do autovetor. Com o método PROMETHEE II, nenhum procedimento é fornecido para determinar os pesos. O método TODIM apresenta uma diretriz de definição de pesos, porém também deixa livre para o usuário escolher um procedimento para definir os pesos. Portanto, tanto o PROMETHEE II quanto o TODIM permitem a obtenção de pesos de critérios pré-definidos por outros métodos MCDM. Nesta pesquisa, os autovetores calculados pelo método AHP serviram como base para avaliar os pesos dos critérios, padronizando os dados para a aplicação dos três métodos multicritérios.

Com relação à frequência dos métodos na literatura, observou-se por meio da análise bibliométrica que o método AHP é a ferramenta mais citada na área de tomada de decisão, sendo assim o método preferido dos autores em relação a todas outras metodologias estabelecidas. O método TODIM e o PROMETHEE são pouco explorados se comparados ao AHP, ocorrendo em menos de $4 \%$ de todos os artigos pesquisados.

A disponibilidade de ferramentas de software para os métodos AHP e PROMETHEE II é uma vantagem em relação ao TODIM, pois com a utilização de um software, os vários procedimentos de cálculos ficam encobertos, facilitando e agilizando a obtenção de uma solução. Até o término deste trabalho, não foi localizado qualquer ferramenta de software disponível baseada no método TODIM. Também é importante ressaltar que, todos os três métodos são aplicáveis utilizando-se de uma planilha do Microsoft Excel, contudo, neste caso, é recomendável a compreensão do mecanismo de funcionamento dos métodos.

Além das constatações realizadas na presente pesquisa, também se pressupõe outras evidências anteriormente previstas a respeito dos métodos MCDM comparados.

O método AHP é uma ferramenta de decisão útil para consolidar julgamentos de avaliações, já que fornece um procedimento para verificar a consistência de critérios e alternativas. Apesar da sua inerente simplicidade e capacidade para julgar fatores quantitativos e qualitativos, diferentes tipos de críticas foram discutidos na literatura. Por exemplo, o método foi criticado não só pela possibilidade de exibir inversão de classificação (Belton \& Gear, 1983), mas também pela utilização do método de autovalor para determinar os pesos (Bana e Costa \& Vansnick, 2008). A escala de 1 (um) a 9 (nove) também pode gerar inconsistências. Supondo um critério A duas vezes mais importante que B e este 
cinco vezes mais importante que $C$, isso implicaria que $A$ fosse dez vezes mais importante que $C$, o que não é possível devido à escala instituída pelo método.

No que tange ao método TODIM, a utilização da Teoria das Prospectos como sua base paradigmática é seu componente básico de inovação. Essa teoria busca transformar os julgamentos humanos em valores de utilidade compatíveis com a ideia de aversão ao risco no âmbito dos ganhos e propensão ao risco no domínio das perdas, fato esse que ocorre para a maioria dos seres humanos (Kahneman \& Tversky, 1979). O procedimento de cálculo do método TODIM já contempla a normalização de Belton e Gear (1982), o que minimiza a possibilidade de ocorrência de inversões nas classificações das alternativas.

O método PROMETHEE II tem uma abordagem clara do problema de decisão. Uma das complicações do método PROMETHEE é o requisito da definição das preferências de funções dos critérios. Em aplicações em tempo real, pode ser complicado para o tomador de decisão especificar qual forma de função de preferência é adequada para cada critério, ademais de também determinar os parâmetros envolvidos.

As etapas de cálculo pelo método AHP apresenta o seguinte procedimento: estruturação hierárquica; comparação par a par para indicação das preferências, utilizando-se da escala fundamental do AHP e de matrizes de decisão; determinação do autovetor (peso) de cada matriz; verificação das consistências dos julgamentos; determinação da importância relativa de cada alternativa e classificação das alternativas. Já o método TODIM segue a seguinte diretriz para os cálculos: a formação de uma matriz de desejabilidades; a comparação paritária entre critérios, valorados por meio da escala fundamental de Saaty, seguida do cálculo do critério de referência, associado à teoria dos Prospectos; e o tratamento matemático dos juízos de valor. No tocante a sequência de cálculos do PROMETHEE II, as etapas são: para a sua estruturação, é estabelecida pela identificação das alternativas, seguida pela identificação dos critérios de avaliação das alternativas, seus pesos e sua função de preferência; é desenvolvida a matriz de decisão, seguida pela construção da matriz dos índices de preferência de cada alternativa; e a classificação das alternativas é realizada com base no fluxo líquido, que permite ordenar as alternativas.

\section{Considerações finais}

Esta pesquisa teve como objetivo a comparação de três diferentes métodos de apoio à decisão multicritério: AHP, TODIM e PROMETHEE II. Para se alcançar essa proposta, os métodos foram aplicados a um problema de seleção de software. Com a comparação dos métodos multicritérios ficou claro a importância destas ferramentas no que se refere à tomada de decisões. A vantagem da utilização das 
abordagens é, entre outros fatores, o de oferecer maior transparência e clareza ao processo decisório e na obtenção de uma classificação ordenada.

No entanto, este estudo demonstrou que a aplicação dos métodos AHP, TODIM e PROMETHEE Il pode produzir resultados diferentes, ratificando resultados de pesquisas análogas que testaram e compararam entre si outros métodos MCDM diferentes. Deste modo, com base na literatura e na aplicação dos métodos MCDM utilizados no experimento, este artigo contribui para mostrar que nenhum método MCDM isolado é inequivocamente mais válido que os outros, sendo que cada um tem falhas em potencial. Assim, recomenda-se que, sempre que possível, mais de um método deve ser aplicado ao mesmo problema, a fim de proporcionar uma compreensão de decisão mais abrangente.

Esta pesquisa também traz contribuições para a Engenharia de Produção, no campo de Pesquisa Operacional, apresentando uma percepção da aplicabilidade, complexidade, e laborosidade requerida do usuário quanto à utilização dos métodos AHP, TODIM e PROMETHEE II. Embora os três métodos se baseiem em diferentes paradigmas, a metodologia de aplicação utilizada possibilitou o apontamento de características individuais, pontos positivos e negativos de um método sobre o outro.

Embora os resultados desta comparação em alguns aspectos quantitativos possam ser replicados para outros contextos de problema, a realização de novas comparações usando outros parâmetros relevantes, com distintas quantidades de fornecedores e critérios, além de experimentos com outros métodos MCDM pode trazer novas contribuições à literatura. Por isso, sugere-se que pesquisas futuras sejam realizadas com o foco de ampliar as avaliações aqui feitas de modo a poder obter conclusões mais amplas sobre o tema estudado.

\section{Referências}

Anojkumar, L., Ilangkumaran, M., \& Sasirekha, V. (2014). Comparative analysis of MCDM methods for pipe material selection in sugar industry. Expert systems with applications, 41(6), 2964-2980. DOI: https://doi.org/10.1016/j.eswa.2013.10.028

Bana e Costa, C. A., \& Vansnick, J. C. (2008). A critical analysis of the eigenvalue method used to derive priorities in AHP. European Journal of Operational Research, 187(3), 1422-1428. DOI: https://doi.org/10.1016/j.ejor.2006.09.022

Belton, V., \& Gear, T. (1983). On a short-coming of Saaty's method of analytic hierarchies. Omega, 11(3), 228-230. DOI: https://doi.org/10.1016/0305-0483(83)90047-6

Bhushan, N. e Rai, K. (2004). Strategic decision making: applying the analytic hierarchy process. London; New York: Springer. Disponível em: https://link.springer.com/book/10.1007/b97668

Brans, J. P., \& Mareschal, B. (2005). PROMETHEE methods, in 'Multiple Criteria Decision Analysis: State of the Art Surveys'. Springer, New York, 163-186. DOI: https://doi.org/10.1007/0-387-230815_5 
Dehghani, H., Siami, A., \& Haghi, P. (2017). A new model for mining method selection based on grey and TODIM methods. Journal of Mining and Environment, 8(1), 49-60. DOI: 10.22044/jme.2016.626

Doukas, H., Patlitzianas, K. D., \& Psarras, J. (2006). Supporting sustainable electricity technologies in Greece using MCDM. Resources Policy, 31(2), 129-136. DOI: https://doi.org/10.1016/j.resourpol.2006.09.003

Dulmin, R., \& Mininno, V. (2003). Supplier selection using a multi-criteria decision aid method. Journal of purchasing and supply management, 9(4), 177-187. DOI: 10.1016/S1478-4092(03)00032-3

Efe, B. (2016). An integrated fuzzy multi criteria group decision making approach for ERP system selection. Applied Soft Computing, 38, 106-117. DOI: 10.1016/j.asoc.2015.09.037

Flament, M. (1999). Glosario multicriterio. España: Red Iberoamericana de Evaluación y Decisión Multicriterio. Disponível em: http://www.unesco.org.uy/red-m/glosariom.htm

Gomes, L. F. A. M. (2007). Teoria da decisão. São Paulo: Thomson. Disponível em: https://www.travessa.com.br/teoria-da-decisao-1-ed-2007/artigo/3dff5e01-2a1d-4ce5-ab9c237b8c8d096b

Gomes, L. F. A. M., \& Rangel, L. A. D. (2009). An application of the TODIM method to the multicriteria rental evaluation of residential properties. European Journal of Operational Research, 193(1), 204-211. DOI: https://doi.org/10.1016/j.ejor.2007.10.046

Gurbuz, T., Alptekin, S. E., \& Alptekin, G. I. (2012). A hybrid MCDM methodology for ERP selection problem with interacting criteria. Decision Support Systems, 54(1), 206-214. DOI: https://doi.org/10.1016/j.dss.2012.05.006

Ho, W., Xu, X., \& Dey, P. K. (2010). Multi-criteria decision making approaches for supplier evaluation and selection: A literature review. European Journal of operational research, 202(1), 16-24. DOI: https://doi.org/10.1016/j.ejor.2009.05.009

Hodgett, R. E. (2016). Comparison of multi-criteria decision-making methods for equipment selection. The International Journal of Advanced Manufacturing Technology, 85(5-8), 11451157. DOI: https://doi.org/10.1007/s00170-015-7993-2

Ishizaka, A., \& Labib, A. (2009). Analytic hierarchy process and expert choice: Benefits and limitations. Or Insight, 22(4), 201-220. DOI: https://doi.org/10.1057/ori.2009.10

Ishizaka, A., \& Nemery, P. (2013). Multi-criteria decision analysis: methods and software. John Wiley \& Sons. DOI: 10.1002/9781118644898

Kahneman, D. \& Tversky, A. (1979). Prospect theory: An analysis of decision under risk. Econometrica: Journal of the econometric society, 263-291. DOI: https://doi.org/10.2307/1914185

Kilic, H. S., Zaim, S., \& Delen, D. (2015). Selecting “The Best” ERP system for SMEs using a combination of ANP and PROMETHEE methods. Expert Systems with Applications, 42(5), 2343-2352. DOI: 10.1016/j.eswa.2014.10.034 
Lim, M. C., Ayoko, G. A., Morawska, L., Ristovski, Z. D., \& Jayaratne, E. R. (2007). Influence of fuel composition on polycyclic aromatic hydrocarbon emissions from a fleet of in-service passenger cars. Atmospheric Environment, 41(1), 150-160. DOI: https://doi.org/10.1016/j.atmosenv.2006.07.044

Lima Junior, F. R. (2013). Comparação entre os métodos Fuzzy TOPSIS e Fuzzy AHP no apoio à tomada de decisão para seleção de fornecedores. Master's Dissertation, Escola de Engenharia de São Carlos, University of São Paulo, São Carlos. . Disponível em: https://teses.usp.br/teses/disponiveis/18/18156/tde-12092013103003/publico/FranciscoDEFINITIVO.pdf

Longaray, A. A., POPIOLEK Jr, T. L., Munhoz, P. R., Geri, F. S., \& Castelli, T. M. (2015). Caracterização da produção científica brasileira sobre a aplicação de métodos multicritério de apoio à decisão: uma análise das publicações entre 2004-2013. XXXV Encontro Nacional de Engenharia de Produção. DOI: https://doi.org/10.47059/geintecmagazine.v7i3.949

Muralidharan, C., Anantharaman, N., \& Deshmukh, S. G. (2002). A multi-criteria group decision making model for supplier rating. Journal of supply chain management, 38(3), 22-33. DOI: https://doi.org/10.1111/j.1745-493X.2002.tb00140.x

Noronha, S. M. D. (1998). Um modelo multicritérios para apoiar a decisão da escolha do combustível para alimentação de caldeiras usadas na indústria têxtil. Dissertação (Mestrado em Engenharia de Produção), Florianópolis, UFSC. Disponível em: https://repositorio.ufsc.br/xmlui/bitstream/handle/123456789/77755/139137.pdf?sequence $=1 \&$ isAllowed=y

Önüt, S., Kara, S. S., \& Işik, E. (2009). Long term supplier selection using a combined fuzzy MCDM approach: A case study for a telecommunication company. Expert systems with applications, 36(2), 3887-3895. DOI: 10.1016/j.eswa.2008.02.045

Padovani, M., Carvalho, M. M. D., \& Muscat, A. R. N. (2010). Seleção e alocação de recursos em portfólio de projetos: estudo de caso no setor químico. Gestão \& Produção, 17(1), 157-180. DOI: 10.1590/S0104-530X2010000100013

Passos, A. C., \& Gomes, L. F. A. M. (2005). Enfoque multicritério à teoria das prospectivas: fundamentos e aplicação. Revista de Administração Mackenzie (Mackenzie Management Review), 6(1). Disponível em: http://www.spell.org.br/documentos/ver/11485/enfoquemulticriterio-a-teoria-das-prospectivas-fundamentos-e-aplicacao/i/pt-br

Qaradaghi, M. (2016). Investigation of Multi-Criteria Decision Consistency: A Triplex Approach to Optimal Oilfield Portfolio Investment Decisions (Doctoral dissertation, The George Washington University). Disponível em: https://www.proquest.com/openview/7702f4e396c67c5fc525be195b9f8a39/1?pqorigsite $=$ gscholar $\& c b l=18750$

Rangel, L. A. D., \& Gomes, L. F. A. M. (2007). Determinação do valor de referência do aluguel de imóveis residenciais empregando o método TODIM. Pesquisa Operacional, 27(2), 357-372. DOI: 10.1590/S0101-74382007000200009

Ribeiro, L. D. S., Passos, A. C., \& Teixeira, M. G. (2012). Seleção de tecnologias de comunicações no exército brasileiro utilizando os métodos multicritério de análise hierárquica, TODIM e 
software Sapiens. Production, 22(1), 132-141. DOI: http://dx.doi.org/10.1590/S010365132011005000063

Saaty, T. L. (1980). The Analytic Hierarchy Process: Planning, Priority Setting, Resources Allocation. McGraw: New York. DOI: https://doi.org/10.1016/0377-2217(82)90022-4

Selmi, M., Kormi, T., \& Ali, N. B. H. (2013). Comparing multi-criteria decision aid methods through a ranking stability index. International Conference on Modeling, Simulation and Applied Optimization, IEEE, 1-5. DOI: 10.1109/ICMSAO.2013.6552593

Sen, D. K., Datta, S., \& Mahapatra, S. S. (2016). Application of TODIM (Tomada de Decisión Inerativa Multicritero) for industrial robot selection. Benchmarking: An International Journal, 23(7), 1818-1833. DOI: 10.1108/BIJ-07-2015-0078

Trendowicz, A. \& Kopczyńska, S. (2014). Adapting Multi-Criteria Decision Analysis for Assessing the Quality of Software Products. Current Approaches and Future Perspectives. Advances in Computers. 93, 153-226. DOI: https://doi.org/10.1016/B978-0-12-800162-2.00004-X

Zindani, D., Maity, S. R., Bhowmik, S., \& Chakraborty, S. (2017). A material selection approach using the TODIM (Tomada de Decisao Interativa Multicriterio) method and its analysis. International Journal of Materials Research, 108(5), 345-354. DOI: 10.3139/146.111489 